\title{
Estimation of AR (2) Model with Dependent Errors for Unbounded Stationary and Nonstationary Time Series
}

\author{
Prof. Ahmed Amin EL-Sheikh \\ Professor of Statistics \\ Dept. of Applied Statistics and \\ Econometrics, Faculty of Graduate Studies \\ for Statistical Research, Cairo University \\ Aham103@yahoo.com
}

\author{
Mohammed Ahmed Farouk Ahmed \\ Lecturer of Statistics \\ High Institute of Computer and Information \\ Technology \\ Al-Shorouk Academy, Cairo \\ M.arslan2030@gmail.com
}

\begin{abstract}
In this paper the GLS and the ML estimators, the variance-covariance matrix, the unbiased for the GLS and the ML estimators of parameters of AR (2) model with constant in case of dependent errors have been derived, the simulation results shown that the values of MSE and Thiel's $U$ in case of unbounded stationary time series for all sample size $\mathrm{T}$ are less than the values of MSE and Thiel's U in case of unbounded nonstationary time series which approved that the results for unbounded stationary times series are better than the results for unbounded nonstationary times series, and the simulation results for unbounded nonstationary time series shown that by using the measurement of MSE the best case among of all cases of nonstationary which gives the smallest values of MSE is case four when the first and the second conditions of stationary conditions for AR (2) model are exists, while by using the measurement of Thiel's U the best case among of all cases of nonstationary which gives the smallest values of Thiel's $U$ is case six when the second and the third conditions of stationary conditions for AR (2) model are exists.
\end{abstract}

Keywords: AR (2) Model; GLS Estimators; ML Estimators; Mean Squared Error; Thiel's Inequality Coefficient; Unbounded Stationary Time Series; Unbounded Nonstationary Time Series. 


\section{Introduction}

The classical regression model seeks to determine the relationship between the dependent variable and the independent variables. This regression model could be simple or multiple. However, in the linear regression model, certain assumptions are made on how a dataset will be produced by an underlying data-generating process. According to Greene (2002), these assumptions include linearity, homoscedasticity, normality, and no autocorrelation between the error terms. Moreover, the regression model describes the value of the dependent variable as the sum of two parts, a deterministic part, and a random part.

The error term is primarily a disturbance to an already stable relationship and can capture the remaining information in the dependent variable which could not be explained by the independent variables. Relating to the assumption on the error term, if the assumption of no correlation in the error term is violated, then, the underlying model would be rendered invalid with the standard errors of the parameters becoming biased. Moreover, if the errors are correlated, the least-squares estimators are inefficient and the estimated variances are not appropriate Granger and Newbold (1974) and Akpan, et al (2016).

By definition, autocorrelation is the lag correlation of a given series with itself, lagged by some time units Gujarati (2004). Thus, when applying regression models to economic/management data in the presence of autocorrelation, the ordinary least squares estimation method ceases to provide efficient estimators and appropriate variances.

The analysis of time series is very important and it is a rapidly evolving field, generally, time series is a sequence of values a specific variable has taken on over some time. The observations have a natural ordering in time. Usually, if a series of observations are referred to as a time series then some regularity of the observation frequency was assumed. Of course, the observation frequency could be more frequent than yearly. For instance, observations may be available for each quarter, each month, or even each day of a particular period. Nowadays, time series of stock prices or other financial market variables are even available at a much higher frequency such as every few minutes or seconds, Lütkepohl and Krätzig (2004).

A model often specified for the generation of an economic time series is the stochastic difference equation with independently and identically distributed errors. In practice, the most 
common assumption is that the time series is stationary. However, there are situations in which the stationarity assumption is not appropriate. Two such situations are testing the random walk hypothesis or unit root hypothesis and testing the first difference hypothesis, Evans and Savin (1981).

For the second-order autoregressive AR (2) model in the case of real roots, the stationarity conditions for an AR (2) processes are as follows:

$$
\begin{aligned}
& \text { 1) } \rho_{1}+\rho_{2}<1 \\
& \text { 2) } \rho_{2}-\rho_{1}<1 \\
& \text { 3) }\left|\rho_{2}\right|<1
\end{aligned}
$$

where $\rho_{1}$ and $\rho_{2}$ are the autoregressive coefficients of the AR (2) model, David (2012)

Autoregressive time series with a unit root has been the subject of much recent attention in the econometrics literature. In part, this is because the unit root hypothesis is of considerable interest in applications, not only with data from financial and commodity markets where it has a long history but also with aggregate time series, Phillips (1987).

In an attempt to overcome the weaknesses of the ordinary least squares estimation method in the presence of autocorrelation, this study seeks to apply the generalized least squares estimation method since the least-squares estimation method does not make use of the information of the unexplained variance as captured by the error terms in the dependent variable, whereas the generalized least squares (GLS) takes such information, the unexplained variance into account explicitly and is accomplished, Akpan and Moffat (2018).

In this paper, the GLS and the ML estimators, the variance-covariance matrix for the GLS and ML estimators of parameters of AR (2) model with constant in case of dependent errors will be derived and a simulation study in two cases unbounded stationary time series and unbounded nonstationary time series will be derived.

\section{Model and Assumptions}

The second-order autoregressive AR (2) with a constant model in case of dependent errors takes the following form:

$$
y_{t}=\alpha+\rho_{1} y_{t-1}+\rho_{2} y_{t-2}+u_{t}, \quad t=1, \ldots, T,
$$


where $y_{t}$ is time series, $T$ is the sample size, $y_{0}=y_{-1}=0, u_{t}$ are dependent error terms $\rho_{1}$ and $\rho_{2}$ are the autoregressive coefficients, and $\alpha$ is the constant term.

Model (2) can be represented in matrix form as follows:

$$
Y=X \beta+\mathbf{u}
$$

Where:

$$
\beta=\left[\begin{array}{c}
\alpha \\
\rho_{1} \\
\rho_{2}
\end{array}\right], X=\left[\begin{array}{ccc}
1 & y_{0} & y_{-1} \\
1 & y_{1} & y_{0} \\
1 & y_{2} & y_{1} \\
\vdots & \vdots & \vdots \\
1 & y_{T-1} & y_{T-2}
\end{array}\right], Y=\left[\begin{array}{c}
y_{1} \\
y_{2} \\
y_{3} \\
\vdots \\
y_{T}
\end{array}\right] \text { and } \mathbf{u}=\left[\begin{array}{c}
u_{1} \\
u_{2} \\
u_{3} \\
\vdots \\
u_{T}
\end{array}\right]
$$

By assuming that $u_{t}$ are dependent error terms which generating by the first-order stationary autoregressive process AR (1) model then, it will be as follows:

$u_{t}=\phi_{1} u_{t-1}+e_{t},\left|\phi_{1}\right|<1, t=1, \ldots, T$

Where the error terms $e_{t}$ are i.i.d. $N\left(0, \sigma_{e}^{2}\right)$ and achieved the following assumptions:

$$
\left.\begin{array}{l}
\text { 1) } E\left(e_{t}\right)=0 \quad \forall t \\
\text { 2) } \sigma_{e}^{2}=E\left(e_{t}^{2}\right)=\sigma^{2} \quad \forall t \\
\text { 3) } \sigma_{s t}=\operatorname{Cov}\left(e_{t}, e_{s}\right)=E\left(e_{t} e_{s}\right)=0 \quad \forall t \neq s
\end{array}\right\}
$$

To the sample interval $t=1, \ldots, T$ the variance-covariance matrix for the vector of error terms $\mathbf{u}=\left[u_{1}, u_{2}, \ldots, u_{T}\right]$ for model (2) can be obtained by using the lag operator $(L)$ as follows:

From equation (5) $u_{t}=\phi_{1} u_{t-1}+e_{t}$ then:

$$
\begin{aligned}
& u_{t}-\phi_{1} u_{t-1}=e_{t} \\
& \left(1-\phi_{1} L\right) u_{t}=e_{t} \\
& u_{t}=\left(1-\phi_{1} L\right)^{-1} e_{t}=\sum_{j=0}^{\infty} \phi_{1}^{j} L^{j} e_{t}=\sum_{j=0}^{\infty} \phi_{1}^{j} e_{t-j},\left|\phi_{1}\right|<1
\end{aligned}
$$

Then $E\left(u_{t}\right), \operatorname{Var}\left(u_{t}\right)$ and $\operatorname{Cov}\left(u_{t}, u_{t-s}\right)$ will be as follows: 
i) $E\left(u_{t}\right)=0$

i i ) $\operatorname{Var}\left(u_{t}\right)=\frac{\sigma^{2}}{\left(1-\phi_{1}^{2}\right)}$

iii) $\operatorname{Cov}\left(u_{t}, u_{t-s}\right)=\sigma^{2} \phi_{1}^{s}\left(\frac{1}{1-\phi_{1}^{2}}\right), s=1,2,3, \ldots$

Then, by using equations (9) and (10), the variance-covariance matrix for the vector of error terms $\mathbf{u}=\left[u_{1}, u_{2}, \ldots, u_{T}\right]$ will be:

$\operatorname{Cov}(\mathbf{u})=E\left\{\left[\begin{array}{c}u_{1} \\ u_{2} \\ \vdots \\ u_{T}\end{array}\right]\left[\begin{array}{lllll}u_{1} & u_{2} & u_{3} & \cdots & u_{T}\end{array}\right]\right\}=\sigma^{2} \Omega$

Where:

$$
\Omega=\frac{1}{\left(1-\phi_{1}^{2}\right)}\left[\begin{array}{ccccc}
1 & \phi_{1} & \phi_{1}^{2} & \cdots & \phi_{1}^{T-1} \\
\phi_{1} & 1 & \phi_{1} & \cdots & \phi_{1}^{T-2} \\
\phi_{1}^{2} & \phi_{1} & 1 & \cdots & \phi_{1}^{T-3} \\
\vdots & \vdots & \vdots & & \vdots \\
\phi_{1}^{T-1} & \phi_{1}^{T-2} & \phi_{1}^{T-3} & \cdots & 1
\end{array}\right]
$$

Fox and Weisberg (2018)

\section{GLS Estimation for AR (2) Model in Case of Dependent Errors}

In this section, the GLS estimators, unbiased of GLS estimators and the variancecovariance matrix for GLS estimators for the parameters of AR (2) model with constant in case of dependent errors will be derived.

\subsection{The GLS Estimators for the Parameters of AR (2) Model}

The GLS estimators for the parameters of AR (2) with a constant model in case of dependent errors as in equation (3) will be derived according to the following lemma:

Lemma (1): The GLS estimators for the parameters of AR (2) with a constant model in case of dependent errors as in equation (3) under the assumptions of the model will be as follows: 


$$
\begin{aligned}
& \tilde{\alpha}=\frac{K_{11} N_{11}+K_{12} N_{12}+K_{13} N_{13}}{G} \\
& \tilde{\rho}_{1}=\frac{K_{21} N_{11}+K_{22} N_{12}+K_{23} N_{13}}{G} \\
& \tilde{\rho}_{2}=\frac{K_{31} N_{11}+K_{32} N_{12}+K_{33} N_{13}}{G}
\end{aligned}
$$

Where:

$$
\begin{aligned}
& K_{11}=C F-E^{2}, K_{22}=\Delta F-B^{2}, K_{33}=\Delta C-A^{2}, K_{12}=K_{21}=B E-A F \\
& K_{32}=K_{23}=A B-\Delta E \text { and } K_{13}=K_{31}=A E-C B \\
& G=\Delta C F-\Delta E^{2}-A^{2} F+2 A B E-B^{2} C \\
& \Delta=1-\phi_{1}+1-2 \phi_{1}+\phi_{1}^{2}+1-2 \phi_{1}+\phi_{1}^{2}+\cdots+1-\phi_{1}=T-2(T-1) \phi_{1}+(T-2) \phi_{1}^{2} \\
& A=\left(1-\phi_{1}\right) y_{0}+\left(1-2 \phi_{1}+\phi_{1}^{2}\right) y_{1}+\left(1-2 \phi_{1}+\phi_{1}^{2}\right) y_{2}+\cdots+\left(1-\phi_{1}\right) y_{T-1} \\
& =\left(1-\phi_{1}\right) y_{0}+\left(1-\phi_{1}\right) y_{T-1}+\left(1-2 \phi_{1}+\phi_{1}^{2}\right) \sum_{t=1}^{T-2} y_{t} \\
& =\left[y_{-1}-\phi_{1} y_{0}\right] y_{1}+\left[y_{T-3}-\phi_{1} y_{T-2}\right] y_{T}+\sum_{t=1}^{T-2}\left[-\phi_{1}\left(y_{t-2}+y_{t}\right)+\left(1+\phi_{1}^{2}\right) y_{t-1}\right] y_{t+1} \\
& B=\left(1-\phi_{1}\right) y_{-1}+\left(1-2 \phi_{1}+\phi_{1}^{2}\right) y_{0}+\left(1-2 \phi_{1}+\phi_{1}^{2}\right) y_{1}+\cdots+\left(1-\phi_{1}\right) y_{T-2} \\
& =\left(1-\phi_{1}\right) y_{-1}+\left(1-\phi_{1}\right) y_{T-2}+\left(1-2 \phi_{1}+\phi_{1}^{2}\right) \sum_{t=1}^{T-2} y_{t-1} \\
& C=\left(y_{0}-\phi_{1} y_{1}\right) y_{0}+\left[-\phi_{1}\left(y_{0}+y_{2}\right)+\left(1+\phi_{1}^{2}\right) y_{1}\right] y_{1}+\left[-\phi_{1}\left(y_{1}+y_{3}\right)+\left(1+\phi_{1}^{2}\right) y_{2}\right] y_{2} \\
& +\cdots+\left[y_{T-2}-\phi_{1} y_{T-1}\right] y_{T-1} \\
& =\left(y_{0}-\phi_{1} y_{1}\right) y_{0}+\left[y_{T-2}-\phi_{1} y_{T-1}\right] y_{T-1}+\sum_{t=1}^{T-2}\left[-\phi_{1}\left(y_{t-1}+y_{t+1}\right)+\left(1+\phi_{1}^{2}\right) y_{t}\right] y_{t} \\
& E=\left(y_{0}-\phi_{1} y_{1}\right) y_{-1}+\left[-\phi_{1}\left(y_{0}+y_{2}\right)+\left(1+\phi_{1}^{2}\right) y_{1}\right] y_{0}+\left[-\phi_{1}\left(y_{1}+y_{3}\right)+\left(1+\phi_{1}^{2}\right) y_{2}\right] y_{1} \\
& +\cdots+\left[y_{T-2}-\phi_{1} y_{T-1}\right] y_{T-2} \\
& =\left(y_{0}-\phi_{1} y_{1}\right) y_{-1}+\left[y_{T-2}-\phi_{1} y_{T-1}\right] y_{T-2}+\sum_{t=1}^{T-2}\left[-\phi_{1}\left(y_{t-1}+y_{t+1}\right)+\left(1+\phi_{1}^{2}\right) y_{t}\right] y_{t-1} \\
& F=\left(y_{-1}-\phi_{1} y_{0}\right) y_{-1}+\left[-\phi_{1}\left(y_{-1}+y_{1}\right)+\left(1+\phi_{1}^{2}\right) y_{0}\right] y_{0}+\left[-\phi_{1}\left(y_{0}+y_{2}\right)+\left(1+\phi_{1}^{2}\right) y_{1}\right] y_{1} \\
& +\cdots+\left[y_{T-3}-\phi_{1} y_{T-2}\right] y_{T-2} \\
& =\left(y_{-1}-\phi_{1} y_{0}\right) y_{-1}+\left[y_{T-3}-\phi_{1} y_{T-2}\right] y_{T-2}+\sum_{t=1}^{T-2}\left[-\phi_{1}\left(y_{t-2}+y_{t}\right)+\left(1+\phi_{1}^{2}\right) y_{t-1}\right] y_{t-1} \\
& N_{11}=\left(1-\phi_{1}\right) y_{1}+\left(1-2 \phi_{1}+\phi_{1}^{2}\right) y_{2}+\left(1-2 \phi_{1}+\phi_{1}^{2}\right) y_{3}+\cdots+\left(1-\phi_{1}\right) y_{T} \\
& =\left(1-\phi_{1}\right) y_{1}+\left(1-\phi_{1}\right) y_{T}+\left(1-2 \phi_{1}+\phi_{1}^{2}\right) \sum_{t=1}^{T-2} y_{t+1} \\
& N_{12}=\left[y_{0}-\phi_{1} y_{1}\right] y_{1}+\left[-\phi_{1}\left(y_{0}+y_{2}\right)+\left(1+\phi_{1}^{2}\right) y_{1}\right] y_{2}+\left[-\phi_{1}\left(y_{1}+y_{3}\right)+\left(1+\phi_{1}^{2}\right) y_{2}\right] y_{3} \\
& +\cdots+\left[y_{T-2}-\phi_{1} y_{T-1}\right] y_{T} \\
& =\left[y_{0}-\phi_{1} y_{1}\right] y_{1}+\left[y_{T-2}-\phi_{1} y_{T-1}\right] y_{T}+\sum_{t=1}^{T-2}\left[-\phi_{1}\left(y_{t-1}+y_{t+1}\right)+\left(1+\phi_{1}^{2}\right) y_{t}\right] y_{t+1}
\end{aligned}
$$




$$
\begin{aligned}
N_{13}= & {\left[y_{-1}-\phi_{1} y_{0}\right] y_{1}+\left[-\phi_{1}\left(y_{-1}+y_{1}\right)+\left(1+\phi_{1}^{2}\right) y_{0}\right] y_{2}+\left[-\phi_{1}\left(y_{0}+y_{2}\right)+\left(1+\phi_{1}^{2}\right) y_{1}\right] y_{3} } \\
& +\cdots+\left[y_{T-3}-\phi_{1} y_{T-2}\right] y_{T} \\
= & {\left[y_{-1}-\phi_{1} y_{0}\right] y_{1}+\left[y_{T-3}-\phi_{1} y_{T-2}\right] y_{T}+\sum_{t=1}^{T-2}\left[-\phi_{1}\left(y_{t-2}+y_{t}\right)+\left(1+\phi_{1}^{2}\right) y_{t-1}\right] y_{t+1} }
\end{aligned}
$$

Proof:

The GLS Estimator $\widetilde{\beta}$, in general, is defined as follows:

$$
\widetilde{\beta}=\left(X^{\prime} \Omega^{-1} X\right)^{-1} X^{\prime} \Omega^{-1} Y
$$

Fox and Weisberg (2018)

Where for model in equation (3),

$$
\widetilde{\beta}=\left[\begin{array}{c}
\widetilde{\alpha} \\
\widetilde{\rho}_{1} \\
\widetilde{\rho}_{2}
\end{array}\right], X=\left[\begin{array}{ccc}
1 & y_{0} & y_{-1} \\
1 & y_{1} & y_{0} \\
\vdots & \vdots & \vdots \\
1 & y_{T-1} & y_{T-2}
\end{array}\right], Y=\left[\begin{array}{c}
y_{1} \\
y_{2} \\
\vdots \\
y_{T}
\end{array}\right]
$$

By substituting from equations (12) and (14) in equation (13) then the GLS estimator $\tilde{\beta}$ for the model in equation (3) will be as follows:

$$
\begin{aligned}
& {\left[\begin{array}{c}
\tilde{\alpha} \\
\tilde{\rho}_{1} \\
\tilde{\rho}_{2}
\end{array}\right]=\left\{\left[\begin{array}{ccccc}
1 & 1 & 1 & \cdots & 1 \\
y_{0} & y_{1} & y_{2} & \cdots & y_{T-1} \\
y_{-1} & y_{0} & y_{1} & \cdots & y_{T-2}
\end{array}\right]\left(\frac{1}{\left(1-\phi_{1}^{2}\right)}\left[\begin{array}{ccccc}
1 & \phi_{1} & \phi_{1}^{2} & \cdots & \phi_{1}^{T-1} \\
\phi_{1} & 1 & \phi_{1} & \cdots & \phi_{1}^{T-2} \\
\phi_{1}^{2} & \phi_{1} & 1 & \cdots & \phi_{1}^{T-3} \\
\vdots & \vdots & \vdots & & \vdots \\
\phi_{1}^{T-1} & \phi_{1}^{T-2} & \phi_{1}^{T-3} & \cdots & 1
\end{array}\right]\right)^{-1}\left[\begin{array}{ccc}
1 & y_{0} & y_{-1} \\
1 & y_{1} & y_{0} \\
1 & y_{2} & y_{1} \\
\vdots & \vdots & \vdots \\
1 & y_{T-1} & y_{T-2}
\end{array}\right]\right\}^{-1}} \\
& {\left[\begin{array}{lllll}
1 & 1 & 1 & \cdots & 1 \\
y_{0} & y_{1} & y_{2} & \cdots & y_{T-1} \\
y_{-1} & y_{0} & y_{1} & \cdots & y_{T-2}
\end{array}\right]\left(\frac{1}{\left(1-\phi_{1}^{2}\right)}\left[\begin{array}{ccccc}
1 & \phi_{1} & \phi_{1}^{2} & \cdots & \phi_{1}^{T-1} \\
\phi_{1} & 1 & \phi_{1} & \cdots & \phi_{1}^{T-2} \\
\phi_{1}^{2} & \phi_{1} & 1 & \cdots & \phi_{1}^{T-3} \\
\vdots & \vdots & \vdots & & \vdots \\
\phi_{1}^{T-1} & \phi_{1}^{T-2} & \phi_{1}^{T-3} & \cdots & 1
\end{array}\right]\right)^{-1}\left[\begin{array}{l}
y_{1} \\
y_{2} \\
y_{3} \\
\vdots \\
y_{T}
\end{array}\right]}
\end{aligned}
$$

Then, 


$$
\begin{aligned}
& {\left[\begin{array}{c}
\tilde{\alpha} \\
\widetilde{\rho}_{1} \\
\widetilde{\rho}_{2}
\end{array}\right]=\left\{\begin{array}{ccc}
\Delta & A & B \\
A & C & E \\
B & E & F
\end{array}\right\}^{-1}\left[\begin{array}{c}
N_{11} \\
N_{12} \\
N_{13}
\end{array}\right]} \\
& \tilde{\alpha}=\frac{K_{11} N_{11}+K_{12} N_{12}+K_{13} N_{13}}{G} \\
& \widetilde{\rho}_{1}=\frac{K_{21} N_{11}+K_{22} N_{12}+K_{23} N_{13}}{G} \\
& \widetilde{\rho}_{2}=\frac{K_{31} N_{11}+K_{32} N_{12}+K_{33} N_{13}}{G}
\end{aligned}
$$

Where:

$\Delta, A, B, C, E, F, G, N_{11}, N_{12}, N_{13}, K_{11}, K_{22}, K_{33}, K_{12}=K_{21}, K_{32}=K_{23}$ and $K_{13}=K_{31}$ are defined as in lemma (1).

\subsection{The Unbiased for GLS Estimators of Parameters of AR (2) Model}

From equation (13) the GLS estimators for parameters of AR (2) model with constant in case of dependent errors is as follows:

$$
\widetilde{\beta}=\left(X^{\prime} \Omega^{-1} X\right)^{-1} X^{\prime} \Omega^{-1} Y
$$

Then,

$$
\begin{aligned}
E(\widetilde{\beta}) & =\left(X^{\prime} \Omega^{-1} X\right)^{-1} X^{\prime} \Omega^{-1} E(Y) \\
& =\left(X^{\prime} \Omega^{-1} X\right)^{-1} X^{\prime} \Omega^{-1} E\left(X^{\prime} \beta+\mathbf{u}\right) \\
& =\left(X^{\prime} \Omega^{-1} X\right)^{-1} X^{\prime} \Omega^{-1} X^{\prime} \beta+E(\mathbf{u})=\beta+E(\mathbf{u})
\end{aligned}
$$

By using equation (8) then,

$$
E(\widetilde{\beta})=\beta+E(\mathbf{u})=\beta+\mathbf{0}=\beta
$$

Therefore the GLS estimators $\widetilde{\beta}$ are unbiased estimators for $\beta$.

\subsection{Variance-Covariance Matrix for GLS Estimators for AR (2) Model}

In this section the variance-covariance matrix for GLS estimators $\widetilde{\beta}$ of parameters of AR (2) model with constant in case of dependent errors will be obtained according to the following lemma:

Lemma (2): The variance-covariance matrix for GLS estimators $\widetilde{\beta}$ of parameters of AR (2) model with constant in case of dependent errors as in equation (3) under the assumptions of the model will be as follows: 


$\left[\begin{array}{ccc}V(\tilde{\alpha}) & \operatorname{Cov}\left(\tilde{\alpha}, \tilde{\rho}_{1}\right) & \operatorname{Cov}\left(\tilde{\alpha}, \tilde{\rho}_{2}\right) \\ \operatorname{Cov}\left(\tilde{\alpha}, \tilde{\rho}_{1}\right) & V\left(\tilde{\rho}_{1}\right) & \operatorname{Cov}\left(\tilde{\rho}_{1}, \tilde{\rho}_{2}\right) \\ \operatorname{Cov}\left(\tilde{\alpha}, \tilde{\rho}_{2}\right) & \operatorname{Cov}\left(\tilde{\rho}_{1}, \tilde{\rho}_{2}\right) & V\left(\tilde{\rho}_{2}\right)\end{array}\right]=\frac{\sigma^{2}}{G}\left[\begin{array}{ccc}K_{11} & K_{12} & K_{13} \\ K_{21} & K_{22} & K_{23} \\ K_{31} & K_{32} & K_{33}\end{array}\right]$

Where:

$$
\begin{aligned}
& K_{11}=C F-E^{2}, \quad K_{22}=\Delta F-B^{2}, K_{33}=\Delta C-A^{2}, K_{12}=K_{21}=B E-A F \\
& K_{32}=K_{23}=A B-\Delta E \text { and } K_{13}=K_{31}=A E-C B, G=\Delta C F-\Delta E^{2}-A^{2} F+2 A B E-B^{2} C \\
& \Delta=1-\phi_{1}+1-2 \phi_{1}+\phi_{1}^{2}+1-2 \phi_{1}+\phi_{1}^{2}+\cdots+1-\phi_{1}=T-2(T-1) \phi_{1}+(T-2) \phi_{1}^{2} \\
& A=\left(1-\phi_{1}\right) y_{0}+\left(1-2 \phi_{1}+\phi_{1}^{2}\right) y_{1}+\left(1-2 \phi_{1}+\phi_{1}^{2}\right) y_{2}+\cdots+\left(1-\phi_{1}\right) y_{T-1} \\
&=\left(1-\phi_{1}\right) y_{0}+\left(1-\phi_{1}\right) y_{T-1}+\left(1-2 \phi_{1}+\phi_{1}^{2}\right) \sum_{t=1}^{T-2} y_{t} \\
&= {\left[y_{-1}-\phi_{1} y_{0}\right] y_{1}+\left[y_{T-3}-\phi_{1} y_{T-2}\right] y_{T}+\sum_{t=1}^{T-2}\left[-\phi_{1}\left(y_{t-2}+y_{t}\right)+\left(1+\phi_{1}^{2}\right) y_{t-1}\right] y_{t+1} } \\
& B=\left(1-\phi_{1}\right) y_{-1}+\left(1-2 \phi_{1}+\phi_{1}^{2}\right) y_{0}+\left(1-2 \phi_{1}+\phi_{1}^{2}\right) y_{1}+\cdots+\left(1-\phi_{1}\right) y_{T-2} \\
&=\left(1-\phi_{1}\right) y_{-1}+\left(1-\phi_{1}\right) y_{T-2}+\left(1-2 \phi_{1}+\phi_{1}^{2}\right) \sum_{t=1}^{T-2} y_{t-1} \\
& C=\left(y_{0}-\phi_{1} y_{1}\right) y_{0}+\left[-\phi_{1}\left(y_{0}+y_{2}\right)+\left(1+\phi_{1}^{2}\right) y_{1}\right] y_{1}+\left[-\phi_{1}\left(y_{1}+y_{3}\right)+\left(1+\phi_{1}^{2}\right) y_{2}\right] y_{2} \\
&+\cdots+\left[y_{T-2}-\phi_{1} y_{T-1}\right] y_{T-1} \\
&=\left(y_{0}-\phi_{1} y_{1}\right) y_{0}+\left[y_{T-2}-\phi_{1} y_{T-1}\right] y_{T-1}+\sum_{t=1}^{T-2}\left[-\phi_{1}\left(y_{t-1}+y_{t+1}\right)+\left(1+\phi_{1}^{2}\right) y_{t}\right] y_{t} \\
& E=\left(y_{0}-\phi_{1} y_{1}\right) y_{-1}+\left[-\phi_{1}\left(y_{0}+y_{2}\right)+\left(1+\phi_{1}^{2}\right) y_{1}\right] y_{0}+\left[-\phi_{1}\left(y_{1}+y_{3}\right)+\left(1+\phi_{1}^{2}\right) y_{2}\right] y_{1} \\
&+\cdots+\left[y_{T-2}-\phi_{1} y_{T-1}\right] y_{T-2} \\
&=\left(y_{0}-\phi_{1} y_{1}\right) y_{-1}+\left[y_{T-2}-\phi_{1} y_{T-1}\right] y_{T-2}+\sum_{t=1}^{T-2}\left[-\phi_{1}\left(y_{t-1}+y_{t+1}\right)+\left(1+\phi_{1}^{2}\right) y_{t}\right] y_{t-1} \\
& F=\left(y_{-1}-\phi_{1} y_{0}\right) y_{-1}+\left[-\phi_{1}\left(y_{-1}+y_{1}\right)+\left(1+\phi_{1}^{2}\right) y_{0}\right] y_{0}+\left[-\phi_{1}\left(y_{0}+y_{2}\right)+\left(1+\phi_{1}^{2}\right) y_{1}\right] y_{1} \\
&+\cdots+\left[y_{T-3}-\phi_{1} y_{T-2}\right] y_{T-2} \\
&=\left(y_{-1}-\phi_{1} y_{0}\right) y_{-1}+\left[y_{T-3}-\phi_{1} y_{T-2}\right] y_{T-2}+\sum_{t=1}^{T-2}\left[-\phi_{1}\left(y_{t-2}+y_{t}\right)+\left(1+\phi_{1}^{2}\right) y_{t-1}\right] y_{t-1} \\
& P r
\end{aligned}
$$

\section{Proof:}

The variance-covariance matrix for GLS estimators in general is as follows:

$$
V(\widetilde{\beta})=\sigma^{2}\left(X^{\prime} \Omega^{-1} X\right)^{-1}
$$

Fox and Weisberg (2018)

Where for model in equation (3), 


$$
\begin{aligned}
& V(\widetilde{\beta})=\left[\begin{array}{ccc}
V(\tilde{\alpha}) & \operatorname{Cov}\left(\tilde{\alpha}, \widetilde{\rho}_{1}\right) & \operatorname{Cov}\left(\tilde{\alpha}, \widetilde{\rho}_{2}\right) \\
\operatorname{Cov}\left(\tilde{\alpha}, \widetilde{\rho}_{1}\right) & V\left(\widetilde{\rho}_{1}\right) & \operatorname{Cov}\left(\widetilde{\rho}_{1}, \widetilde{\rho}_{2}\right) \\
\operatorname{Cov}\left(\tilde{\alpha}, \widetilde{\rho}_{2}\right) & \operatorname{Cov}\left(\widetilde{\rho}_{1}, \widetilde{\rho}_{2}\right) & V\left(\widetilde{\rho}_{2}\right)
\end{array}\right], X=\left[\begin{array}{ccc}
1 & y_{0} & y_{-1} \\
1 & y_{1} & y_{0} \\
1 & y_{2} & y_{1} \\
\vdots & \vdots & \vdots \\
1 & y_{T-1} & y_{T-2}
\end{array}\right], \\
& \Omega=\frac{1}{\left(1-\phi_{1}^{2}\right)}\left[\begin{array}{ccccc}
1 & \phi_{1} & \phi_{1}^{2} & \cdots & \phi_{1}^{T-1} \\
\phi_{1} & 1 & \phi_{1} & \cdots & \phi_{1}^{T-2} \\
\phi_{1}^{2} & \phi_{1} & 1 & \cdots & \phi_{1}^{T-3} \\
\vdots & \vdots & \vdots & & \vdots \\
\phi_{1}^{T-1} & \phi_{1}^{T-2} & \phi_{1}^{T-3} & \cdots & 1
\end{array}\right]
\end{aligned}
$$

By substituting from equation (17) in equation (16) then the $V(\tilde{\beta})$ for model in equation (3) will be as follows:

$$
\begin{aligned}
& {\left[\begin{array}{ccc}
V(\tilde{\alpha}) & \operatorname{Cov}\left(\tilde{\alpha}, \widetilde{\rho}_{1}\right) & \operatorname{Cov}\left(\tilde{\alpha}, \widetilde{\rho}_{2}\right) \\
\operatorname{Cov}\left(\tilde{\alpha}, \widetilde{\rho}_{1}\right) & V\left(\widetilde{\rho}_{1}\right) & \operatorname{Cov}\left(\widetilde{\rho}_{1}, \widetilde{\rho}_{2}\right) \\
\operatorname{Cov}\left(\tilde{\alpha}, \widetilde{\rho}_{2}\right) & \operatorname{Cov}\left(\widetilde{\rho}_{1}, \widetilde{\rho}_{2}\right) & V\left(\widetilde{\rho}_{2}\right)
\end{array}\right]=} \\
& \sigma^{2}\left\{\left[\begin{array}{ccccc}
1 & 1 & 1 & \cdots & 1 \\
y_{0} & y_{1} & y_{2} & \cdots & y_{T-1} \\
y_{-1} & y_{0} & y_{1} & \cdots & y_{T-2}
\end{array}\right]\left(\frac{1}{\left(1-\phi_{1}^{2}\right)}\left[\begin{array}{ccccc}
1 & \phi_{1} & \phi_{1}^{2} & \cdots & \phi_{1}^{T-1} \\
\phi_{1} & 1 & \phi_{1} & \cdots & \phi_{1}^{T-2} \\
\phi_{1}^{2} & \phi_{1} & 1 & \cdots & \phi_{1}^{T-3} \\
\vdots & \vdots & \vdots & & \vdots \\
\phi_{1}^{T-1} & \phi_{1}^{T-2} & \phi_{1}^{T-3} & \cdots & 1
\end{array}\right]\right)^{-1}\left[\begin{array}{ccc}
1 & y_{0} & y_{-1} \\
1 & y_{1} & y_{0} \\
1 & y_{2} & y_{1} \\
1 & \vdots & \vdots \\
1 & y_{T-1} & y_{T-2}
\end{array}\right]\right\}
\end{aligned}
$$

Then,

$\left[\begin{array}{ccc}V(\tilde{\alpha}) & \operatorname{Cov}\left(\tilde{\alpha}, \tilde{\rho}_{1}\right) & \operatorname{Cov}\left(\tilde{\alpha}, \tilde{\rho}_{2}\right) \\ \operatorname{Cov}\left(\tilde{\alpha}, \tilde{\rho}_{1}\right) & V\left(\tilde{\rho}_{1}\right) & \operatorname{Cov}\left(\tilde{\rho}_{1}, \tilde{\rho}_{2}\right) \\ \operatorname{Cov}\left(\tilde{\alpha}, \tilde{\rho}_{2}\right) & \operatorname{Cov}\left(\tilde{\rho}_{1}, \tilde{\rho}_{2}\right) & V\left(\tilde{\rho}_{2}\right)\end{array}\right]=\sigma^{2}\left\{\begin{array}{ccc}\Delta & A & B \\ A & C & E \\ B & E & F\end{array}\right\}$

$\left[\begin{array}{ccc}V(\tilde{\alpha}) & \operatorname{Cov}\left(\tilde{\alpha}, \tilde{\rho}_{1}\right) & \operatorname{Cov}\left(\tilde{\alpha}, \tilde{\rho}_{2}\right) \\ \operatorname{Cov}\left(\tilde{\alpha}, \tilde{\rho}_{1}\right) & V\left(\tilde{\rho}_{1}\right) & \operatorname{Cov}\left(\tilde{\rho}_{1}, \tilde{\rho}_{2}\right) \\ \operatorname{Cov}\left(\tilde{\alpha}, \tilde{\rho}_{2}\right) & \operatorname{Cov}\left(\tilde{\rho}_{1}, \tilde{\rho}_{2}\right) & V\left(\tilde{\rho}_{2}\right)\end{array}\right]=\frac{\sigma^{2}}{G}\left[\begin{array}{ccc}K_{11} & K_{12} & K_{13} \\ K_{21} & K_{22} & K_{23} \\ K_{31} & K_{32} & K_{33}\end{array}\right]$

Where: 
$\Delta, A, B, C, E, F, G, K_{11}, K_{22}, K_{33}, K_{12}=K_{21}, K_{32}=K_{23}$ and $K_{13}=K_{31}$ are defined as in lemma (2).

\section{The ML Estimation for AR (2) Model in Case of Dependent Errors}

In this section, the ML estimators, unbiased of ML estimators and the variance-covariance matrix for ML estimators for the parameters of AR (2) model with constant in case of dependent errors will be derived.

\subsection{The ML Estimators for the Parameters of AR (2) Model}

The ML estimators for the parameters of AR (2) with a constant model in case of dependent errors as in equation (3) will be derived according to the following lemma:

Lemma (3): The ML estimators for the parameters of AR (2) with a constant model in case of dependent errors as in equation (3) under the assumptions of the model will be as the GLS estimators as in lemma (1).

\section{Proof:}

From equations (8) and (11) then,

$$
\mathbf{u} \sim N_{T}\left(0, \sigma^{2} \Omega\right)
$$

From equations (3) and (18) then,

$$
Y \sim N_{T}\left(X \beta, \sigma^{2} \Omega\right)
$$

By using equation (19) then,

$$
\mathrm{f}\left(Y ; X, \beta, \sigma^{2}\right)=\left(2 \pi \sigma^{2}\right)^{-\frac{T}{2}}|\Omega|^{-\frac{1}{2}} \exp \left[-\frac{1}{2 \sigma^{2}}(Y-X \beta)^{\prime} \Omega^{-1}(Y-X \beta)\right]
$$

By using equation (20) then the log of the maximum likelihood function for model in equation (3) will be as follows:

$$
\begin{aligned}
& \ln L\left(\beta, \sigma^{2} \mid Y, X\right)= \\
& \quad-\frac{T}{2} \ln 2 \pi-\frac{T}{2} \ln \sigma^{2}-\frac{1}{2} \ln |\Omega|-\frac{1}{2 \sigma^{2}}(Y-X \beta)^{\prime} \Omega^{-1}(Y-X \beta)
\end{aligned}
$$

Fox and Weisberg (2018)

And to obtain the ML estimator $\hat{\beta}$ the following normal equation must be solved 


$$
\frac{\partial \ln L\left(\beta, \sigma^{2} \mid Y, X\right)}{\partial \beta}=-\frac{1}{2 \hat{\sigma}_{g}^{2}}\left[-X^{\prime} \Omega^{-1} Y+2\left(X^{\prime} \Omega^{-1} X\right) \hat{\beta}\right]=\mathbf{0}
$$

By solving equation (22) the ML estimator $\hat{\beta}$ will be as follows:

$$
\hat{\beta}=\left(X^{\prime} \Omega^{-1} X\right)^{-1} X^{\prime} \Omega^{-1} Y
$$

The form ML estimators $\hat{\beta}$ in equation (23) is the same form as GLS estimators $\widetilde{\beta}$ in equation (13) and by substituting from equations (4) and (12) in equation (23) then the ML estimators $\hat{\beta}$ for AR (2) model with constant in case of dependent errors will be as GLS estimators $\widetilde{\beta}$ in equation (15) and hence the ML estimators $\hat{\beta}$ are unbiased estimators for $\beta$.

\subsection{Variance-Covariance Matrix for the ML Estimators for AR (2) Model}

In this section the variance-covariance matrix for ML estimators $\hat{\beta}$ of parameters of AR (2) model with constant in case of dependent errors will be obtained according to the following lemma:

Lemma (4): The variance-covariance matrix for ML estimators $V(\hat{\beta})$ of parameters of AR (2) model with constant in case of dependent errors as in equation (3) under the assumptions of the model will be as the variance-covariance matrix for GLS estimators $V(\widetilde{\beta})$ as in lemma (2).

\section{Proof:}

The variance-covariance matrix for ML estimators, in general, is as follows:

$$
V(\hat{\beta})=\sigma^{2}\left(X^{\prime} \Omega^{-1} X\right)^{-1}
$$

Amer (2015)

Where for model in equation (3), 


$$
\begin{aligned}
& V(\hat{\beta})=\left[\begin{array}{ccc}
V(\hat{\alpha}) & \operatorname{Cov}\left(\hat{\alpha}, \hat{\rho}_{1}\right) & \operatorname{Cov}\left(\hat{\alpha}, \hat{\rho}_{2}\right) \\
\operatorname{Cov}\left(\hat{\alpha}, \hat{\rho}_{1}\right) & V\left(\hat{\rho}_{1}\right) & \operatorname{Cov}\left(\hat{\rho}_{1}, \hat{\rho}_{2}\right) \\
\operatorname{Cov}\left(\hat{\alpha}, \hat{\rho}_{2}\right) & \operatorname{Cov}\left(\hat{\rho}_{1}, \hat{\rho}_{2}\right) & V\left(\hat{\rho}_{2}\right)
\end{array}\right], X=\left[\begin{array}{ccc}
1 & y_{0} & y_{-1} \\
1 & y_{1} & y_{0} \\
1 & y_{2} & y_{1} \\
\vdots & \vdots & \vdots \\
1 & y_{T-1} & y_{T-2}
\end{array}\right], \\
& \Omega=\frac{1}{\left(1-\phi_{1}^{2}\right)}\left[\begin{array}{ccccc}
1 & \phi_{1} & \phi_{1}^{2} & \cdots & \phi_{1}^{T-1} \\
\phi_{1} & 1 & \phi_{1} & \cdots & \phi_{1}^{T-2} \\
\phi_{1}^{2} & \phi_{1} & 1 & \cdots & \phi_{1}^{T-3} \\
\vdots & \vdots & \vdots & & \vdots \\
\phi_{1}^{T-1} & \phi_{1}^{T-2} & \phi_{1}^{T-3} & \cdots & 1
\end{array}\right]
\end{aligned}
$$

By substituting from equation (25) in equation (24) then the variance-covariance matrix for ML estimators $V(\hat{\beta})$ for the model in equation (3) will be as the variance-covariance matrix for GLS estimators $V(\tilde{\beta})$ as in lemma (2).

\section{Simulation Study}

In this section a simulation study is used to obtain bias, MSE, Thiel's U for GLS and ML estimators of AR (2) with a constant model in case of dependent errors under two cases the first case for unbounded stationary time series and the second case for unbounded nonstationary time series as follows:

\subsection{Unbounded Stationary Time Series}

A simulation study is used to obtain bias, MSE, Thiel's U for GLS and ML estimators of AR (2) model with constant in case of dependent errors which obtained in equation (15) for stationary time series by using initial values for $\rho_{1}=0.95$ and $\rho_{2}=0.03$ for which the three conditions for stationarity in equation (1) exist in case of five samples size $\mathrm{T}=30,50,100,200$ and 500 and ten values for the coefficient of dependent errors $\phi_{1}= \pm 0.5, \pm 0.4, \pm 0.3, \pm 0.2$ and \pm 0.1 by 5000 replications and the following results can be discussed for the five cases:

Case (1): $\mathbf{T}=\mathbf{3 0}$

It can be noticed from table (1) in the appendix of tables that in general the values of MSE for positive values of $\phi_{1}$ are more than the values of MSE for negative values of $\phi_{1}$, the values of Thiel's $U$ for positive values of $\phi_{1}$ are less than the values of Thiel's $U$ for negative values of $\phi_{1}$, 
the values of MSE fluctuated with the descending values of $\phi_{1}$, and the values of Thiel's U are ascending with the descending values of $\phi_{1}$.

\section{Case (2): $\mathbf{T}=\mathbf{5 0}$}

It can be noticed from the table (1) in the appendix of tables for sample size $\mathrm{T}=50$ that in general the values of MSE for positive values of $\phi_{1}$ are more than the values of MSE for negative values of $\phi_{1}$, the values of Thiel's U for positive values of $\phi_{1}$ are less than the values of Thiel's U for negative values of $\phi_{1}$, the values of MSE fluctuated with the descending values of $\phi_{1}$, and the values of Thiel's $U$ are ascending with the descending values of $\phi_{1}$.

\section{Case (3): $\mathbf{T}=100$}

It can be noticed from the table (1) in the appendix of tables for sample size $\mathrm{T}=100$ that in general the values of MSE for positive values of $\phi_{1}$ are more than the values of MSE for negative values of $\phi_{1}$, the values of Thiel's U for positive values of $\phi_{1}$ are less than the values of Thiel's U for negative values of $\phi_{1}$, the values of MSE fluctuated with the descending values of $\phi_{1}$, and the values of Thiel's $U$ are ascending with the descending values of $\phi_{1}$.

\section{Case (4): $T=200$}

It can be noticed from the table (1) in the appendix of tables for sample size $\mathrm{T}=200$ that in general the values of MSE for positive values of $\phi_{1}$ are more than the values of MSE for negative values of $\phi_{1}$, the values of Thiel's $U$ for positive values of $\phi_{1}$ are less than the values of Thiel's U for negative values of $\phi_{1}$, the values of MSE fluctuated with the descending values of $\phi_{1}$, and the values of Thiel's $U$ are ascending with the descending values of $\phi_{1}$.

\section{Case (5): $\mathbf{T}=\mathbf{5 0 0}$}

It can be noticed from the table (1) in the appendix of tables for sample size $\mathrm{T}=500$ that in general the values of MSE for positive values of $\phi_{1}$ are more than the values of MSE for negative values of $\phi_{1}$, the values of Thiel's U for positive values of $\phi_{1}$ are less than the values of Thiel's U for negative values of $\phi_{1}$, the values of MSE fluctuated with the descending values of $\phi_{1}$, and the values of Thiel's $U$ are ascending with the descending values of $\phi_{1}$. 


\subsection{Unbounded Nonstationary Time Series}

A simulation study is used to obtain bias, MSE, Thiel's U for GLS and ML estimators of AR (2) model with constant in case of dependent errors which obtained in equation (15) for nonstationary time series by using six pairs of initial values for $\rho_{1}$ and $\rho_{2}$ for which the three conditions for stationarity in equation (1) are non-exists in various ways in case of five sample size $\mathrm{T}=30,50,100,200$ and 500 and ten values for the coefficient of dependent errors $\phi_{1}= \pm 0.5, \pm 0.4, \pm 0.3, \pm 0.2$ and \pm 0.1 by 5000 replications. The following results can be discussed for the next six cases:

\section{Case 1: All Conditions of Stationary are Non-exists for Unbounded Time Series}

In this case, the initial values for $\rho_{1}$ and $\rho_{2}$ are $\rho_{1}=1.2$ and $\rho_{2}=2.3$ which make all conditions of stationarity conditions for AR (2) model in equation (1) are non-exists and for unbounded time series, it can be noticed from the table (2) in the appendix of tables that for all sample sizes the values of MSE for positive values of $\phi_{1}$ are more than the values of MSE for negative values of $\phi_{1}$, the values of Thiel's $U$ for positive values of $\phi_{1}$ are less than the values of Thiel's $U$ for negative values of $\phi_{1}$, for sample sizes, $T=30,50$ and 100 in case of positive values of $\phi_{1}$ the values of MSE fluctuated with the descending values of $\phi_{1}$, for sample sizes, T $=500,200$ and 100 in case of negative values of $\phi_{1}$ the values of MSE are descending with the descending values of $\phi_{1}$ and for all sample sizes the values of Thiel's $U$ are ascending with the descending values of $\phi_{1}$.

\section{CASE 2: The First Condition of Stationary Conditions is Exists}

In this case, the initial values for $\rho_{1}$ and $\rho_{2}$ are $\rho_{1}=-2$ and $\rho_{2}=1.1$ which make the first condition of stationarity conditions for AR (2) model in equation (1) exists and for unbounded time series, it can be noticed from the table (3) in the appendix of tables that for all sample sizes the values of MSE for positive values of $\phi_{1}$ are more than the values of MSE for negative values of $\phi_{1}$, for sample size T $=30$ the values of Thiel's $U$ for positive values of $\phi_{1}$ are less than the values of Thiel's $U$ for negative values of $\phi_{1}$, for sample sizes $T=50,100,200$ 
and 500 the values of Thiel's $U$ for positive values of $\phi_{1}$ are more than the values of Thiel's U for negative values of $\phi_{1}$, for all sample sizes the values of MSE are descending with the descending values of $\phi_{1}$, for sample sizes, $T=30$ the values of Thiel's $U$ are ascending with the descending values of $\phi_{1}$ and for sample sizes T $=50,100,200$ and 500 the values of Thiel's U are descending with the descending values of $\phi_{1}$.

\section{CASE 3: The Second Condition of Stationary Conditions is Exists}

In this case, the initial values for $\rho_{1}$ and $\rho_{2}$ are $\rho_{1}=2.3$ and $\rho_{2}=1.2$ which make the second condition of stationarity conditions for AR (2) model in equation (1) exists and for unbounded time series, it can be noticed from the table (4) in the appendix of tables that for all sample sizes the values of MSE for positive values of $\phi_{1}$ are more than the values of MSE for negative values of $\phi_{1}$, for all sample sizes the values of Thiel's $U$ for positive values of $\phi_{1}$ are more than the values of Thiel's $U$ for negative values of $\phi_{1}$, for sample sizes, $\mathrm{T}=30$ and 50 for positive values of $\phi_{1}$ the values of MSE fluctuated with the descending values of $\phi_{1}$, for sample sizes $\mathrm{T}=30$ and 50 for negative values of $\phi_{1}$ the values of MSE are descending with the descending values of $\phi_{1}$, for sample sizes, T = 100, 200 and 500 the values of MSE are descending with the descending values of $\phi_{1}$ and in general for all sample sizes the values of Thiel's $U$ are descending with the descending values of $\phi_{1}$.

\section{CASE 4: The First and the Second Conditions of Stationary Conditions are Exists}

In this case, the initial values for $\rho_{1}$ and $\rho_{2}$ are $\rho_{1}=1.3$ and $\rho_{2}=-1.5$ which make the first and the second conditions of stationarity conditions for AR (2) model in equation (1) are exists and for unbounded time series, it can be noticed from the table (5) in the appendix of tables that for all sample sizes the values of MSE and Thiel's $U$ for positive values of $\phi_{1}$ are more than the values of MSE and Thiel's $U$ for negative values of $\phi_{1}$ and for all sample sizes the values of MSE and Thiel's U are descending with the descending values of $\phi_{1}$. 


\section{CASE 5: The First and the Third Conditions of Stationary Conditions are Exists}

In this case, the initial values for $\rho_{1}$ and $\rho_{2}$ are $\rho_{1}=-1.7$ and $\rho_{2}=0.8$ which make the first and the third conditions of stationarity conditions for AR (2) model in equation (1) are exists and for unbounded time series, it can be noticed from the table (6) in the appendix of tables that for all sample sizes the values of MSE for positive values of $\phi_{1}$ are more than the values of MSE for negative values of $\phi_{1}$, for sample size $\mathrm{T}=30$ the values of Thiel's $\mathrm{U}$ for positive values of $\phi_{1}$ are less than the values of Thiel's $U$ for negative values of $\phi_{1}$, for sample sizes $T=50,100$, 200 and 500 the values of Thiel's $U$ for positive values of $\phi_{1}$ are more than the values of Thiel's U for negative values of $\phi_{1}$, for all sample sizes the values of MSE are descending with the descending values of $\phi_{1}$, for sample size $\mathrm{T}=30$ the values of Thiel's $U$ are ascending with the descending values of $\phi_{1}$ and for sample sizes $\mathrm{T}=50,100,200$ and 500 the values of Thiel's $U$ are descending with the descending values of $\phi_{1}$.

\section{CASE 6: The Second and the Third Conditions of Stationary Conditions are Exists}

In this case, the initial values for $\rho_{1}$ and $\rho_{2}$ are $\rho_{1}=1.7$ and $\rho_{2}=0.1$ which make the second and the third conditions of stationarity conditions for AR (2) model in equation (1) are exists and for unbounded time series, it can be noticed from the table (7) in the appendix of tables that for all sample sizes the values of MSE and Thiel's $U$ for positive values of $\phi_{1}$ are less than the values of MSE and Thiel's $U$ for negative values of $\phi_{1}$, for sample size $\mathrm{T}=30$ and 50 the values of MSE are fluctuate with the descending values of $\phi_{1}$, for sample size T $=100,200$ and 500 MSE are descending with the descending values of $\phi_{1}$, for all sample size and positive values of $\phi_{1}$ the values of Thiel's $U$ are descending with the descending values of $\phi_{1}$ and for all sample size and negative values of $\phi_{1}$ the values of Thiel's $U$ are ascending with the descending values of $\phi_{1}$. 


\section{Conclusion}

- The GLS and the ML estimators, the unbiased and the variance-covariance matrix for the GLS and the ML estimators of parameters of AR (2) model with constant in case of dependent errors have been derived.

- For unbounded stationary time series and by using the measurements of MSE and Thiel's U the best case among all cases of unbounded stationary time series which gives the smallest values of MSE and Thiel's $U$ is case five when the sample size is $\mathrm{T}=500$.

- When all conditions of stationary conditions for the AR (2) model in equation (1) are nonexists the values of MSE fluctuate with the ascending values of sample size $\mathrm{T}$ and the values of Thiel's $U$ are ascending with the ascending values sample size $T$.

- When the first condition of stationary conditions for AR (2) model in equation (1) exists the values of MSE and Thiel's $U$ are ascending with the ascending values sample size $T$.

- When the second condition of stationary conditions for AR (2) model in equation (1) exists the values of MSE and Thiel's $U$ are ascending with the ascending values sample size T.

- When the first and the second conditions of stationary conditions for AR (2) model in equation (1) exists the values of MSE and Thiel's $U$ are ascending with the ascending values of sample size $\mathrm{T}$.

- When the first and the third conditions of stationary conditions for the AR (2) model in equation (1) exists the values of MSE are descending when the sample size increase from $\mathrm{T}=30$ to $\mathrm{T}=50$ and then the values of MSE are ascending with the ascending values of sample size $\mathrm{T}$ and the values of Thiel's $U$ are ascending with the ascending values sample size $T$.

- When the second and the third conditions of stationary conditions for AR (2) model in equation (1) exists the values of MSE for positive values of $\phi_{1}$ are descending in most cases with the ascending values of sample size T, the values of MSE for negative values of $\phi_{1}$ are ascending with the ascending values of sample size $T$ and for all sample sizes the values of Thiel's $U$ are descending with the ascending values sample size $\mathrm{T}$.

- By using the measurement of MSE the best case among all cases of nonstationary which gives the smallest values of MSE is case four when the first and the second conditions of stationary conditions for AR (2) model in equation (1) exists. 
- By using the measurement of Thiel's U the best case among all cases of nonstationary which gives the smallest values of Thiel's $U$ is case six when the second and the third conditions of stationary conditions for AR (2) model in equation (1) exists.

- Finally by using the measurements of MSE and Thiel's U the results for unbounded stationary time series are best than all cases of unbounded nonstationary time series.

\section{References}

[1] Akpan EA, Moffat IU, Ekpo NB., (2016), "Modeling regression with time series errors of gross domestic product on government expenditure". International Journal of Innovation and Applied Studies, No. 18, V. 4, pp. 990-996.

[2] Amer, G. A., (2015), "Econometrics and Time Series Analysis (Theory, Methods, Applications)" Cairo University.

[3] David E. G., (2012), "Stationarity Conditions for an AR(2) Process" Department of Economics, University of Victoria, Canada. http://www.sfu.ca/ baa7/Teaching/econ818/StationarityAR2.pdf.

[4] Evans, G. B. A., and Savin, N. E., (1981), Testing for Unit Roots, Econometrica, Vol. 49, No. 3, pp. 753-779.

[5] Fox, J. and Weisberg, S., (2018), "Time-Series Regression and Generalized Least Squares in R", https://socialsciences.mcmaster.ca/jfox/Books/Companion/appendices/Appendix-TimeseriesRegression.pdf.

[6] Granger CWJ, Newbold P., (1974), "Spurious regressions in econometrics. Journal of Econometrics, No. 2, Pp:111-120.

[7] Greene WH., (2002), “Econometric analysis”. 5th ed., New York: Prentice-Hall.

[8] Gujarati DN.,( 2004), "Basic econometrics", $4^{\text {th }}$ ed. McGraw-Hill.

[9] Lütkepohl, H. and Krätzig M., (2004), Applied Time Series Econometrics, 1st ed., Cambridge University Press.

[10] Phillips, P.C.B. (1987), " Time series Regression with a Unit Root", Econometrica, Vol. 55, No. 2. pp. 277-302. 


\section{Appendix of Tables}

Results of Bias, MSE, Thiel's U for GLS and ML Estimators of AR (2) Model for

Unbounded Stationary Time Series

Table (1)

\begin{tabular}{|c|c|c|c|c|c|c|c|c|c|c|}
\hline & \multicolumn{5}{|c|}{$\mathbf{T}=\mathbf{3 0}$} & \multicolumn{5}{|c|}{$\mathbf{T}=\mathbf{5 0}$} \\
\hline$\phi_{1}$ & bias $\alpha$ & bias $\rho_{1}$ & bias $\rho_{2}$ & MSE & $\begin{array}{c}\text { Thiel's } \\
\text { U }\end{array}$ & bias $\alpha$ & bias $\rho_{1}$ & bias $\rho_{2}$ & MSE & $\begin{array}{c}\text { Thiel's } \\
\text { U }\end{array}$ \\
\hline 0.5 & -0.162 & -0.426 & 0.298 & $\mathbf{1 7 5 9 . 8 3 7}$ & 0.159 & -0.393 & 0.262 & -0.349 & 3326.305 & 0.112 \\
\hline 0.4 & -0.222 & $\begin{array}{c}-0.653 \\
\end{array}$ & 0.477 & 3545.570 & 0.163 & 0.279 & -0.824 & 0.811 & $\mathbf{5 0 3 7 . 9 9 4}$ & 0.117 \\
\hline 0.3 & 1.119 & -2.239 & 2.546 & 724.385 & 0.170 & -0.092 & 0.085 & -0.180 & 16.671 & 0.122 \\
\hline 0.2 & -0.017 & -0.323 & 0.166 & 614.416 & 0.179 & -0.240 & 0.690 & -0.799 & 4450.855 & 0.130 \\
\hline 0.1 & -0.135 & 0.044 & -0.236 & 93.757 & 0.188 & -0.059 & 0.006 & -0.094 & 37.354 & 0.140 \\
\hline-0.1 & -0.121 & -0.061 & -0.092 & 32.165 & 0.213 & -0.083 & -0.044 & -0.044 & 2.346 & 0.163 \\
\hline-0.2 & -0.116 & -0.120 & -0.043 & 29.371 & 0.228 & -0.087 & -0.034 & -0.054 & 1.315 & 0.177 \\
\hline-0.3 & -0.005 & -0.200 & 0.035 & 106.404 & 0.248 & -0.082 & -0.052 & -0.036 & 1.331 & 0.194 \\
\hline-0.4 & -0.086 & 0.020 & -0.186 & 40.620 & 0.270 & -0.088 & -0.046 & -0.041 & 1.280 & 0.214 \\
\hline-0.5 & -0.079 & -0.090 & -0.067 & 19.521 & 0.297 & -0.087 & -0.055 & -0.032 & 1.335 & 0.238 \\
\hline & \multicolumn{5}{|c|}{$T=100$} & \multicolumn{5}{|c|}{$T=200$} \\
\hline$\phi_{1}$ & bias $\alpha$ & bias $\rho_{1}$ & bias $\rho_{2}$ & MSE & $\begin{array}{c}\text { Thiel's } \\
\text { U }\end{array}$ & bias $\alpha$ & bias $\rho_{1}$ & bias $\rho_{2}$ & MSE & $\begin{array}{c}\text { Thiel's } \\
\text { U }\end{array}$ \\
\hline 0.5 & -0.123 & 0.266 & -0.307 & 144.171 & 0.071 & -0.100 & 0.017 & -0.023 & 5.339 & 0.046 \\
\hline 0.4 & -0.116 & 0.091 & -0.128 & 55.385 & 0.076 & -0.096 & 0.063 & -0.070 & 3.267 & 0.051 \\
\hline 0.3 & -0.171 & 0.159 & -0.191 & 75.671 & 0.082 & -0.102 & 0.009 & -0.016 & 3.156 & 0.057 \\
\hline 0.2 & -0.092 & 0.018 & -0.052 & 1.518 & 0.089 & -0.100 & 0.017 & -0.023 & 1.434 & 0.062 \\
\hline 0.1 & -0.093 & 0.006 & -0.039 & 1.102 & 0.097 & -0.100 & -0.003 & -0.003 & 1.210 & 0.069 \\
\hline-0.1 & -0.093 & -0.016 & -0.017 & 1.043 & 0.117 & -0.098 & -0.006 & -0.0001 & 1.062 & 0.083 \\
\hline-0.2 & -0.093 & -0.020 & -0.012 & 1.067 & 0.128 & -0.098 & -0.009 & 0.003 & 1.083 & 0.091 \\
\hline-0.3 & -0.093 & -0.024 & -0.009 & 1.117 & 0.142 & -0.098 & -0.011 & 0.004 & 1.132 & 0.101 \\
\hline-0.4 & -0.093 & -0.026 & -0.007 & 1.202 & 0.157 & -0.098 & -0.012 & 0.006 & 1.217 & 0.113 \\
\hline-0.5 & -0.093 & -0.027 & -0.006 & 1.337 & 0.176 & -0.098 & -0.013 & 0.007 & 1.351 & 0.127 \\
\hline \multicolumn{6}{|c|}{$T=500$} & & & & & \\
\hline$\phi_{1}$ & bias $\alpha$ & bias $\rho_{1}$ & bias $\rho_{2}$ & MSE & $\begin{array}{c}\text { Thiel's } \\
\text { U }\end{array}$ & & & & & \\
\hline 0.5 & -0.100 & 0.003 & 0.007 & 4.171 & 0.030 & & & & & \\
\hline 0.4 & -0.097 & 0.014 & -0.005 & 1.481 & 0.033 & & & & & \\
\hline 0.3 & -0.097 & 0.006 & 0.003 & 1.309 & 0.036 & & & & & \\
\hline 0.2 & -0.097 & 0.002 & 0.008 & 1.206 & 0.040 & & & & & \\
\hline 0.1 & -0.097 & -0.001 & 0.010 & 1.143 & 0.044 & & & & & \\
\hline-0.1 & -0.098 & -0.004 & 0.013 & 1.101 & 0.053 & & & & & \\
\hline-0.2 & -0.098 & -0.005 & 0.014 & 1.118 & 0.059 & & & & & \\
\hline-0.3 & -0.098 & -0.005 & 0.015 & 1.163 & 0.065 & & & & & \\
\hline-0.4 & -0.0979 & -0.006 & 0.015 & 1.244 & 0.072 & & & & & \\
\hline-0.5 & -0.0978 & -0.006 & 0.015 & 1.376 & 0.082 & & & & & \\
\hline
\end{tabular}


Results of Bias, MSE, Thiel's U for GLS and ML Estimators of AR (2) Model Unbounded Nonstationary Time Series

Table (2)

\begin{tabular}{|c|c|c|c|c|c|c|c|c|c|c|}
\hline & \multicolumn{5}{|c|}{$T=30$} & \multicolumn{5}{|c|}{$\mathbf{T}=\mathbf{5 0}$} \\
\hline$\phi_{1}$ & bias $\alpha$ & bias $\rho_{1}$ & bias $\rho_{2}$ & MSE & $\begin{array}{c}\text { Thiel's } \\
\text { U }\end{array}$ & bias $\alpha$ & bias $\rho_{1}$ & bias $\rho_{2}$ & MSE & $\begin{array}{c}\text { Thiel's } \\
\text { U }\end{array}$ \\
\hline 0.5 & -0.3930 & -0.2840 & -2.3140 & 948.1200 & 0.5020 & -0.3639 & -0.0289 & -2.5501 & 1215.3044 & 0.5208 \\
\hline 0.4 & -0.6761 & -1.0008 & $\begin{array}{l}-1.5711 \\
\end{array}$ & 9624.774 & 0.5013 & 0.5389 & -0.0306 & -2.5418 & 1516.6306 & 0.5213 \\
\hline 0.3 & 1.1390 & -1.3361 & -1.1282 & 12499.37 & 0.5023 & -0.5073 & 2.0572 & -4.6908 & 45514.163 & 0.5209 \\
\hline 0.2 & 0.3485 & -0.3292 & $\begin{array}{l}-2.2769 \\
\end{array}$ & 1919.046 & 0.5029 & -0.6745 & -1.6600 & $\begin{array}{l}-0.8308 \\
\end{array}$ & 10549.906 & 0.5220 \\
\hline 0.1 & -0.1511 & 0.0011 & -2.6021 & 447.7445 & 0.5045 & -0.5379 & -0.4492 & -2.1022 & 1348.4792 & 0.5225 \\
\hline-0.1 & $\begin{array}{l}-0.1289 \\
\end{array}$ & $\begin{array}{l}-0.1691 \\
\end{array}$ & -2.4350 & 455.9769 & 0.5088 & -0.1059 & 0.1901 & -2.7521 & 232.9151 & 0.5251 \\
\hline-0.2 & -0.2416 & 0.0256 & -2.6290 & 235.6406 & 0.5126 & 3.2589 & -6.4286 & 4.0261 & 182656.80 & 0.5276 \\
\hline-0.3 & -0.0704 & -0.0897 & -2.5162 & 93.1722 & 0.5182 & -0.0685 & -0.2280 & -2.3307 & 192.5253 & 0.5310 \\
\hline-0.4 & -0.2977 & -0.1900 & -2.4213 & 244.0119 & 0.5256 & -0.1015 & -0.0797 & -2.4819 & 78.8887 & 0.5358 \\
\hline-0.5 & -0.0894 & -0.0706 & -2.5371 & 50.7405 & 0.5355 & -0.1059 & -0.1039 & -2.4581 & 66.6973 & 0.5424 \\
\hline & \multicolumn{5}{|c|}{$T=100$} & \multicolumn{5}{|c|}{$\mathbf{T}=\mathbf{2 0 0}$} \\
\hline$\phi_{1}$ & bias $\alpha$ & bias $\rho_{1}$ & bias $\rho_{2}$ & MSE & $\begin{array}{c}\text { Thiel's } \\
\text { U }\end{array}$ & bias $\alpha$ & bias $\rho_{1}$ & bias $\rho_{2}$ & MSE & $\begin{array}{c}\text { Thiel's } \\
\text { U }\end{array}$ \\
\hline 0.5 & 0.2121 & -2.0799 & -0.4222 & $\mathbf{8 3 3 5 . 8 2 2}$ & 0.5374 & -0.0449 & -0.1116 & -2.4038 & 2487.4137 & 0.5461 \\
\hline 0.4 & -0.2192 & -0.1568 & -2.3651 & 2645.861 & 0.5375 & $\begin{array}{l}-0.5239 \\
\end{array}$ & 0.1514 & -2.6626 & 2826.6582 & 0.5460 \\
\hline 0.3 & -0.0391 & 0.1103 & -2.6395 & 702.2164 & 0.5373 & -0.1802 & -0.3547 & -2.1569 & 1486.7336 & 0.5460 \\
\hline 0.2 & 0.2535 & 0.5574 & $\begin{array}{l}-3.0919 \\
\end{array}$ & 2804.786 & 0.5370 & -0.0927 & -0.0443 & -2.4714 & 949.8193 & 0.5458 \\
\hline 0.1 & -0.0520 & -0.2773 & -2.2500 & 608.8197 & 0.5374 & -0.1111 & -0.0945 & -2.4210 & 752.5519 & 0.5460 \\
\hline-0.1 & $\begin{array}{l}-0.1033 \\
\end{array}$ & $\begin{array}{l}-0.0849 \\
\end{array}$ & -2.4454 & 246.8071 & 0.5389 & $\begin{array}{l}-0.0923 \\
\end{array}$ & -0.0776 & -2.4378 & \begin{tabular}{|c|}
505.6318 \\
\end{tabular} & 0.5468 \\
\hline-0.2 & $\begin{array}{l}-0.0923 \\
\end{array}$ & $\begin{array}{l}-0.0867 \\
\end{array}$ & -2.4434 & 207.9054 & 0.5401 & $\begin{array}{l}-0.0954 \\
\end{array}$ & $\begin{array}{l}-0.0895 \\
\end{array}$ & -2.4259 & 425.2468 & 0.5474 \\
\hline-0.3 & -0.0956 & -0.0914 & -2.4389 & 177.9503 & 0.5420 & -0.0961 & -0.0941 & -2.4212 & 363.2696 & 0.5484 \\
\hline-0.4 & $\begin{array}{l}-0.0959 \\
\end{array}$ & -0.1045 & -2.4256 & $\mathbf{1 5 4 . 1 0 6 3}$ & 0.5447 & -0.0965 & $\begin{array}{l}-0.0971 \\
\end{array}$ & -2.4182 & 314.3058 & 0.5498 \\
\hline-0.5 & -0.0959 & -0.1105 & -2.4196 & 135.4833 & 0.5486 & -0.0967 & -0.0992 & -2.4161 & 275.1071 & 0.5519 \\
\hline \multicolumn{6}{|c|}{$T=500$} & & & & & \\
\hline$\phi_{1}$ & bias $\alpha$ & bias $\rho_{1}$ & $\operatorname{bias} \rho_{2}$ & MSE & $\begin{array}{c}\text { Thiel's } \\
\text { U }\end{array}$ & & & & & \\
\hline 0.5 & -0.1423 & -0.0890 & -2.4173 & $\mathbf{5 9 9 3 . 4 3 6}$ & 0.5515 & & & & & \\
\hline 0.4 & -0.1027 & -0.0648 & -2.4414 & 4168.803 & 0.5515 & & & & & \\
\hline 0.3 & -0.1093 & -0.0758 & -2.4304 & 3066.791 & 0.5515 & & & & & \\
\hline 0.2 & -0.1083 & -0.0853 & -2.4208 & 2350.387 & 0.5516 & & & & & \\
\hline 0.1 & -0.1075 & -0.0906 & -2.4155 & 1858.778 & 0.5516 & & & & & \\
\hline-0.1 & -0.1062 & -0.0964 & -2.4096 & 1246.458 & 0.5520 & & & & & \\
\hline-0.2 & -0.1057 & -0.0981 & -2.4080 & 1048.376 & 0.5523 & & & & & \\
\hline-0.3 & -0.1053 & -0.0994 & -2.4067 & 894.3062 & 0.5527 & & & & & \\
\hline-0.4 & -0.1049 & -0.1003 & -2.4058 & 772.2282 & 0.5532 & & & & & \\
\hline-0.5 & -0.1046 & -0.1009 & -2.4052 & 674.0333 & 0.5541 & & & & & \\
\hline
\end{tabular}


Table (3)

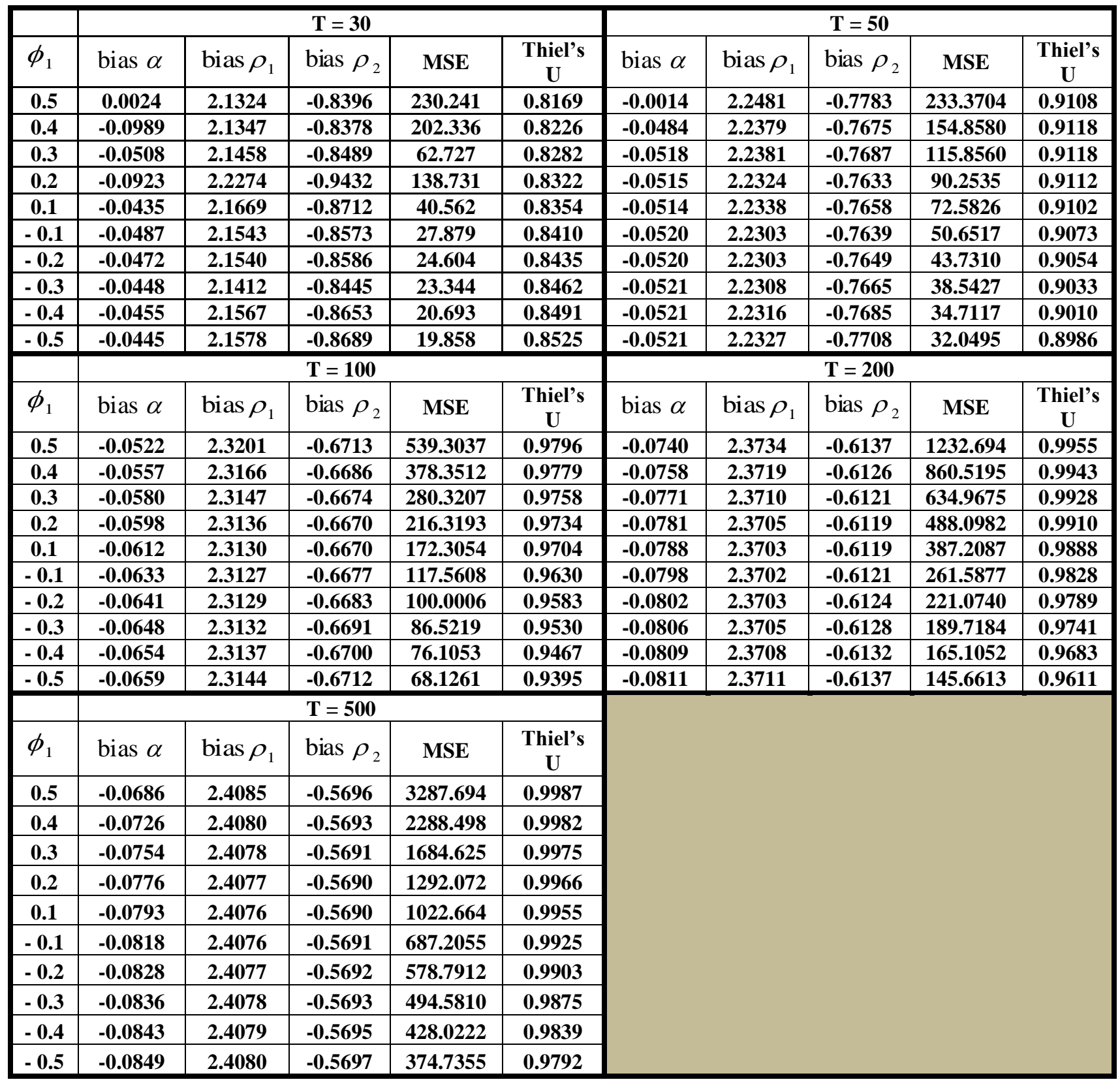


Table (4)

\begin{tabular}{|c|c|c|c|c|c|c|c|c|c|c|}
\hline & \multicolumn{5}{|c|}{$\mathbf{T}=\mathbf{3 0}$} & \multicolumn{5}{|c|}{$\mathbf{T}=\mathbf{5 0}$} \\
\hline$\phi_{1}$ & bias $\alpha$ & bias $\rho_{1}$ & bias $\rho_{2}$ & MSE & $\begin{array}{c}\text { Thiel's } \\
\text { U }\end{array}$ & bias $\alpha$ & bias $\rho_{1}$ & bias $\rho_{2}$ & MSE & $\begin{array}{c}\text { Thiel's } \\
\text { U }\end{array}$ \\
\hline 0.5 & -3.4312 & -2.0387 & -0.6101 & 52310.334 & 0.5103 & 0.1715 & -0.7778 & -1.7753 & 1309.404 & 0.5264 \\
\hline 0.4 & 0.3026 & $\begin{array}{l}-0.5168 \\
\end{array}$ & -2.1562 & 4185.2382 & 0.5094 & -2.8526 & 4.2700 & -7.1237 & 98974.06 & 0.5263 \\
\hline 0.3 & 0.3382 & -1.0543 & $\begin{array}{l}-1.5413 \\
\end{array}$ & 1815.9965 & 0.5091 & 0.1540 & $\begin{array}{l}-0.6343 \\
\end{array}$ & -1.9353 & $\begin{array}{l}756.939 \\
\end{array}$ & 0.5258 \\
\hline 0.2 & $\begin{array}{l}-0.8598 \\
\end{array}$ & -0.1127 & -2.5132 & 3351.6985 & 0.5099 & 0.0618 & -1.6469 & $\begin{array}{l}-0.8783 \\
\end{array}$ & 2653.297 & 0.5260 \\
\hline 0.1 & -0.1705 & -1.5743 & -0.9492 & 3470.1618 & 0.5083 & -0.2321 & -0.6346 & -1.9244 & 489.081 & 0.5237 \\
\hline-0.1 & -0.0286 & -0.5571 & -2.0676 & 141.2536 & 0.5074 & -0.0920 & -0.5358 & -2.0315 & 131.221 & 0.5222 \\
\hline-0.2 & -0.0433 & -0.4830 & -2.1322 & 80.5986 & 0.5068 & -0.0926 & -0.5892 & -1.9749 & 104.950 & 0.5218 \\
\hline-0.3 & -0.0702 & -0.6116 & -2.0029 & 85.2520 & 0.5074 & -0.0982 & -0.6780 & -1.8789 & 145.048 & 0.5222 \\
\hline-0.4 & -0.0826 & -0.6268 & -1.9837 & 51.5509 & 0.5087 & -0.0976 & $\begin{array}{l}-0.6260 \\
\end{array}$ & -1.9371 & 77.477 & 0.5230 \\
\hline-0.5 & -0.0810 & -0.6177 & -1.9914 & 44.4388 & 0.5122 & -0.0961 & $\begin{array}{l}-0.6406 \\
\end{array}$ & -1.9221 & 68.153 & 0.5251 \\
\hline & \multicolumn{5}{|c|}{$T=100$} & \multicolumn{5}{|c|}{$T=200$} \\
\hline$\phi_{1}$ & bias $\alpha$ & bias $\rho_{1}$ & bias $\rho_{2}$ & MSE & $\begin{array}{c}\text { Thiel's } \\
\text { U }\end{array}$ & bias $\alpha$ & $\operatorname{bias} \rho_{1}$ & bias $\rho_{2}$ & MSE & $\begin{array}{c}\text { Thiel's } \\
\text { U }\end{array}$ \\
\hline 0.5 & 0.1785 & -0.4881 & -2.0521 & 2128.389 & 0.5411 & -0.3692 & -0.8298 & -1.6858 & 2710.045 & 0.5482 \\
\hline 0.4 & 0.1540 & -0.3457 & -2.1917 & 2024.014 & 0.5404 & -0.0530 & -0.5453 & -1.9711 & 1795.078 & 0.5478 \\
\hline 0.3 & 0.2050 & -0.4534 & -2.0786 & 1045.529 & 0.5400 & -0.1177 & -0.4910 & -2.0249 & 1263.454 & 0.5475 \\
\hline 0.2 & -0.0423 & -0.5265 & -2.0057 & 487.194 & 0.5392 & -0.0964 & -0.5274 & -1.9884 & 963.029 & 0.5470 \\
\hline 0.1 & -0.0794 & -0.4853 & -2.0478 & 388.224 & 0.5386 & -0.0993 & -0.5704 & -1.9453 & 761.247 & 0.5466 \\
\hline-0.1 & -0.1065 & -0.5826 & -1.9487 & 251.529 & 0.5376 & $\begin{array}{l}-0.0998 \\
\end{array}$ & $\begin{array}{l}-0.5995 \\
\end{array}$ & -1.9160 & 510.920 & 0.5462 \\
\hline-0.2 & -0.1023 & -0.6019 & -1.9291 & 211.630 & 0.5374 & -0.0997 & -0.6061 & -1.9093 & 429.966 & 0.5461 \\
\hline-0.3 & -0.1011 & -0.6127 & -1.9182 & 180.867 & 0.5375 & -0.0997 & -0.6105 & $\begin{array}{l}-1.9049 \\
\end{array}$ & 367.006 & 0.5461 \\
\hline-0.4 & -0.1003 & $\begin{array}{l}-0.6197 \\
\end{array}$ & -1.9111 & 156.594 & 0.5379 & -0.0996 & $\begin{array}{l}-0.6134 \\
\end{array}$ & -1.9020 & 317.140 & 0.5462 \\
\hline-0.5 & -0.0998 & -0.6244 & -1.9063 & 137.191 & 0.5388 & -0.0995 & -0.6154 & -1.9000 & 277.070 & 0.5466 \\
\hline \multicolumn{6}{|c|}{$\mathbf{T}=\mathbf{5 0 0}$} & & & & & \\
\hline$\phi_{1}$ & bias $\alpha$ & $\operatorname{bias} \rho_{1}$ & $\operatorname{bias} \rho_{2}$ & MSE & $\begin{array}{c}\text { Thiel's } \\
\text { U }\end{array}$ & & & & & \\
\hline 0.5 & -0.2784 & -0.2053 & -2.3011 & 6490.813 & 0.5526 & & & & & \\
\hline 0.4 & -0.1004 & -0.5335 & -1.9727 & 4194.880 & 0.5523 & & & & & \\
\hline 0.3 & -0.1039 & -0.5692 & -1.9370 & 3084.210 & 0.5521 & & & & & \\
\hline 0.2 & -0.1039 & -0.5865 & -1.9197 & 2363.296 & 0.5520 & & & & & \\
\hline 0.1 & -0.1039 & -0.5959 & -1.9103 & 1868.674 & 0.5519 & & & & & \\
\hline-0.1 & -0.1036 & -0.6055 & -1.9006 & 1252.574 & 0.5517 & & & & & \\
\hline-0.2 & -0.1034 & -0.6081 & -1.8981 & 1053.210 & 0.5517 & & & & & \\
\hline-0.3 & -0.1032 & -0.6098 & -1.8963 & 898.078 & 0.5517 & & & & & \\
\hline-0.4 & -0.1030 & -0.6111 & -1.8950 & 775.065 & 0.5517 & & & & & \\
\hline-0.5 & -0.1028 & -0.6120 & -1.8941 & 675.981 & 0.5518 & & & & & \\
\hline
\end{tabular}


Table (5)

\begin{tabular}{|c|c|c|c|c|c|c|c|c|c|c|}
\hline & \multicolumn{5}{|c|}{$\mathbf{T}=\mathbf{3 0}$} & \multicolumn{5}{|c|}{$\mathbf{T}=\mathbf{5 0}$} \\
\hline$\phi_{1}$ & bias $\alpha$ & bias $\rho_{1}$ & bias $\rho_{2}$ & MSE & $\begin{array}{c}\text { Thiel's } \\
\text { U }\end{array}$ & bias $\alpha$ & bias $\rho_{1}$ & bias $\rho_{2}$ & MSE & $\begin{array}{c}\text { Thiel's } \\
\text { U }\end{array}$ \\
\hline 0.5 & -0.0288 & -0.6668 & 1.0724 & 21.721 & 0.6990 & -0.0327 & -0.6519 & 1.1622 & 35.704 & 0.7788 \\
\hline 0.4 & -0.0296 & -0.6852 & 1.1087 & 19.671 & 0.6990 & -0.0325 & -0.6532 & 1.1691 & 25.783 & 0.7733 \\
\hline 0.3 & -0.0297 & -0.6634 & 1.0925 & 11.795 & 0.6984 & -0.0330 & -0.6565 & 1.1772 & 19.882 & 0.7677 \\
\hline 0.2 & -0.0296 & -0.6729 & 1.1121 & 9.146 & 0.6969 & -0.0334 & -0.6585 & 1.1829 & 15.868 & 0.7619 \\
\hline 0.1 & -0.0298 & -0.6770 & 1.1234 & 7.760 & 0.6944 & -0.0337 & -0.6598 & 1.1872 & 13.007 & 0.7559 \\
\hline-0.1 & -0.0298 & -0.6766 & 1.1316 & 5.700 & 0.6864 & -0.0340 & -0.6613 & 1.1929 & 9.278 & 0.7427 \\
\hline-0.2 & -0.0299 & -0.6777 & 1.1361 & 5.018 & 0.6808 & -0.0341 & -0.6618 & 1.1948 & 8.019 & 0.7352 \\
\hline-0.3 & -0.0299 & -0.6784 & 1.1393 & 4.465 & 0.6737 & -0.0342 & -0.6622 & 1.1963 & 7.014 & 0.7266 \\
\hline - 0.4 & -0.0299 & -0.6791 & 1.1418 & 4.009 & 0.6649 & -0.0343 & -0.6626 & 1.1974 & 6.200 & 0.7166 \\
\hline-0.5 & -0.0298 & -0.6799 & 1.1437 & 3.630 & 0.6537 & -0.0343 & -0.6631 & 1.1983 & 5.530 & 0.7043 \\
\hline & \multicolumn{5}{|c|}{$T=100$} & \multicolumn{5}{|c|}{$T=200$} \\
\hline$\phi_{1}$ & bias $\alpha$ & bias $\rho_{1}$ & bias $\rho_{2}$ & MSE & $\begin{array}{c}\text { Thiel's } \\
\text { U }\end{array}$ & bias $\alpha$ & bias $\rho_{1}$ & bias $\rho_{2}$ & MSE & $\begin{array}{c}\text { Thiel's } \\
\text { U }\end{array}$ \\
\hline 0.5 & -0.0407 & -0.6213 & 1.2393 & 93.624 & 0.8662 & -0.0443 & -0.5766 & 1.2975 & 248.760 & 0.9212 \\
\hline 0.4 & -0.0412 & -0.6229 & 1.2425 & 66.452 & $\mathbf{0 . 8 5 5 9}$ & -0.0458 & -0.5777 & 1.2989 & 174.158 & 0.9124 \\
\hline 0.3 & -0.0416 & -0.6239 & 1.2449 & 49.733 & 0.8467 & -0.0468 & -0.5784 & 1.2999 & 128.858 & 0.9043 \\
\hline 0.2 & -0.0418 & -0.6246 & 1.2467 & $\mathbf{3 8 . 7 0 4}$ & 0.8382 & -0.0476 & -0.5789 & 1.3007 & 99.284 & 0.8967 \\
\hline 0.1 & -0.0420 & -0.6250 & 1.2480 & 31.038 & 0.8301 & -0.0482 & -0.5792 & 1.3012 & 78.906 & 0.8896 \\
\hline-0.1 & -0.0422 & -0.6255 & 1.2497 & 21.336 & 0.8144 & -0.0491 & -0.5796 & 1.3019 & 53.385 & 0.8759 \\
\hline-0.2 & -0.0423 & -0.6255 & 1.2502 & 18.147 & 0.8062 & -0.0494 & $-\mathbf{- 0 . 5 7 9 7}$ & 1.3022 & 45.079 & 0.8691 \\
\hline-0.3 & -0.0423 & -0.6256 & 1.2505 & 15.641 & 0.7975 & -0.0497 & -0.5798 & 1.3023 & 38.592 & 0.8619 \\
\hline - 0.4 & -0.0424 & -0.6256 & 1.2508 & 13.637 & 0.7877 & -0.0499 & -0.5799 & 1.3025 & 33.427 & 0.8541 \\
\hline-0.5 & -0.0424 & -0.6257 & 1.2509 & 12.007 & 0.7761 & -0.0501 & -0.5800 & 1.3026 & 29.247 & 0.8452 \\
\hline \multicolumn{6}{|c|}{$T=500$} & & & & & \\
\hline$\phi_{1}$ & bias $\alpha$ & bias $\rho_{1}$ & bias $\rho_{2}$ & MSE & $\begin{array}{c}\text { Thiel's } \\
\text { U }\end{array}$ & & & & & \\
\hline 0.5 & -0.0348 & -0.5115 & 1.3724 & 846.788 & 0.9648 & & & & & \\
\hline 0.4 & -0.0400 & -0.5119 & 1.3728 & 589.519 & 0.9595 & & & & & \\
\hline 0.3 & -0.0437 & -0.5122 & 1.3730 & 434.062 & 0.9545 & & & & & \\
\hline 0.2 & -0.0465 & -0.5124 & 1.3732 & 332.988 & 0.9497 & & & & & \\
\hline 0.1 & -0.0486 & -0.5126 & 1.3734 & 263.585 & 0.9451 & & & & & \\
\hline - 0.1 & -0.0518 & -0.5128 & 1.3736 & 177.047 & 0.9361 & & & & & \\
\hline-0.2 & -0.0530 & -0.5129 & 1.3736 & 149.004 & 0.9316 & & & & & \\
\hline-0.3 & -0.0540 & -0.5129 & 1.3737 & 127.156 & 0.9269 & & & & & \\
\hline-0.4 & -0.0549 & -0.5130 & 1.3737 & 109.803 & 0.9220 & & & & & \\
\hline-0.5 & -0.0556 & -0.5131 & 1.3738 & 95.789 & 0.9165 & & & & & \\
\hline
\end{tabular}


Table (6)

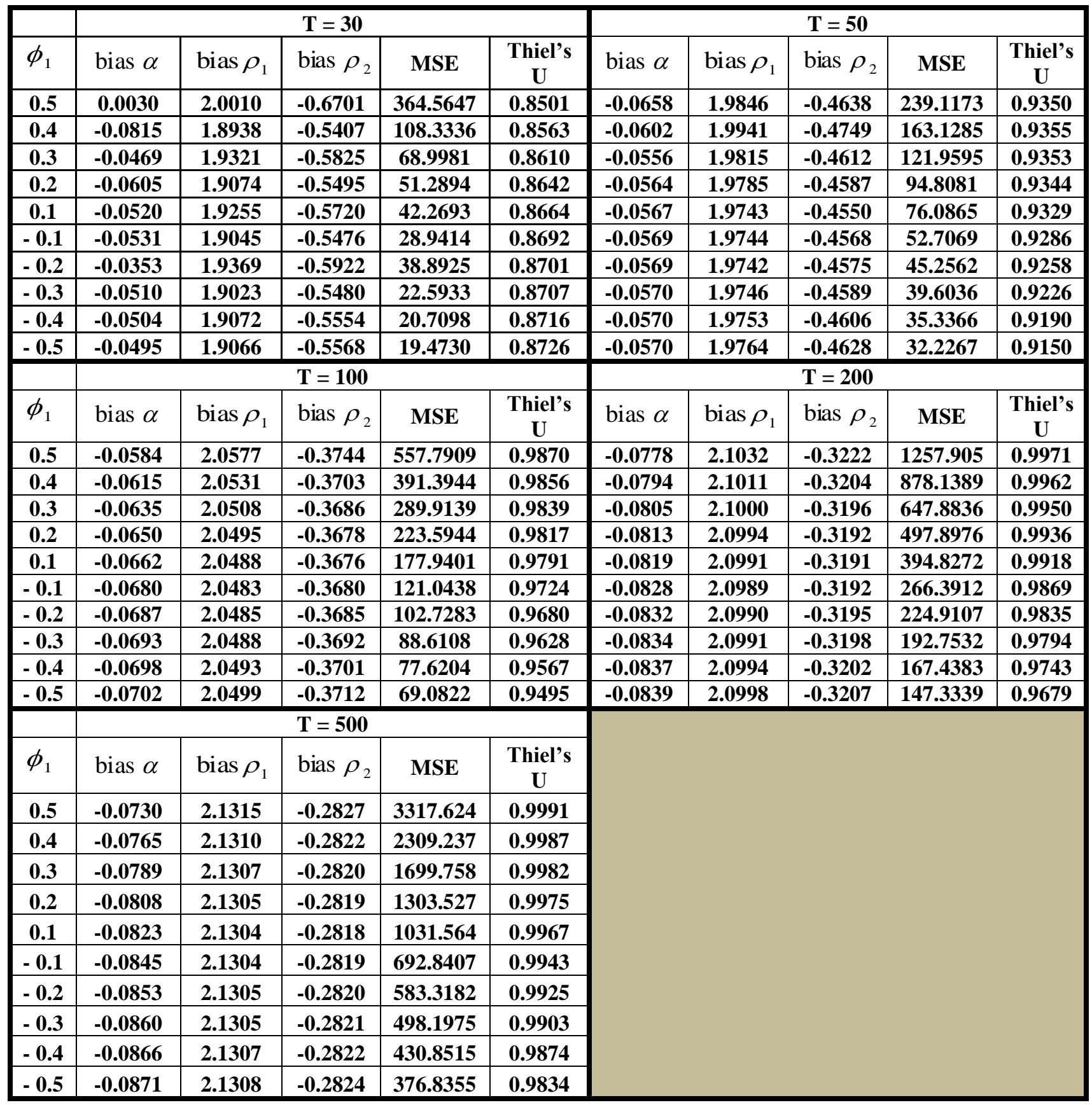


Table (7)

\begin{tabular}{|c|c|c|c|c|c|c|c|c|c|c|}
\hline & \multicolumn{5}{|c|}{$\mathbf{T}=\mathbf{3 0}$} & \multicolumn{5}{|c|}{$\mathbf{T}=\mathbf{5 0}$} \\
\hline$\phi_{1}$ & bias $\alpha$ & bias $\rho_{1}$ & bias $\rho_{2}$ & MSE & $\begin{array}{c}\text { Thiel's } \\
\text { U }\end{array}$ & bias $\alpha$ & $\operatorname{bias} \rho_{1}$ & bias $\rho_{2}$ & MSE & $\begin{array}{c}\text { Thiel's } \\
\text { U }\end{array}$ \\
\hline 0.5 & -4.7120 & 9.4870 & -10.5890 & 5.62652. & 0.3080 & -0.0509 & -1.0110 & 0.1533 & 2407.577 & 0.2988 \\
\hline 0.4 & -0.1759 & -0.6813 & $\begin{array}{l}-0.1348 \\
\end{array}$ & 2893.669 & 0.3052 & 0.0426 & -0.4466 & -0.4269 & 638.176 & 0.2947 \\
\hline 0.3 & -0.0886 & -0.2880 & -0.6536 & 231.0125 & 0.3009 & -0.2195 & 0.1964 & $\begin{array}{l}-1.0888 \\
\end{array}$ & 120.148 & 0.2897 \\
\hline 0.2 & -0.2344 & 0.1468 & -1.0912 & 244.2930 & 0.2987 & 0.0335 & -0.1354 & -0.7375 & 151.031 & 0.2879 \\
\hline 0.1 & $\begin{array}{l}-0.0561 \\
\end{array}$ & -0.7915 & -0.1193 & 2036.596 & 0.2958 & -0.0484 & -0.1112 & -0.7689 & 39.295 & 0.2865 \\
\hline-0.1 & -1.7422 & 1.0786 & -1.4115 & 873.6613 & 0.2970 & -0.1063 & -0.1077 & -0.7725 & 20.744 & 0.2890 \\
\hline-0.2 & -0.0427 & -0.1662 & -0.7772 & 20.6669 & 0.3006 & -0.1937 & -0.3566 & -0.5224 & 205.351 & 0.2922 \\
\hline-0.3 & -0.0850 & -0.1686 & -0.7650 & 19.2119 & 0.3058 & -0.1115 & -0.1805 & -0.6972 & 15.132 & 0.2972 \\
\hline-0.4 & -0.0717 & -0.1939 & -0.7400 & 10.8095 & 0.3148 & -0.0909 & -0.2259 & -0.6510 & 9.326 & 0.3045 \\
\hline-0.5 & -0.0820 & -0.2658 & -0.6662 & 6.2102 & 0.3270 & -0.0938 & -0.2375 & -0.6393 & 8.205 & 0.3145 \\
\hline & \multicolumn{5}{|c|}{$T=100$} & \multicolumn{5}{|c|}{$T=200$} \\
\hline$\phi_{1}$ & bias $\alpha$ & $\operatorname{bias} \rho_{1}$ & bias $\rho_{2}$ & MSE & $\begin{array}{c}\text { Thiel's } \\
\text { U }\end{array}$ & bias $\alpha$ & bias $\rho_{1}$ & bias $\rho_{2}$ & MSE & $\begin{array}{c}\text { Thiel's } \\
\text { U }\end{array}$ \\
\hline 0.5 & -0.1013 & -0.3250 & -0.5198 & 1007.701 & 0.2891 & 0.2559 & 0.2215 & -1.0344 & 1558.600 & 0.2865 \\
\hline 0.4 & -0.0668 & -0.0326 & -0.8038 & 244.189 & 0.2868 & -0.1228 & -0.0989 & -0.7195 & 285.099 & 0.2854 \\
\hline 0.3 & -0.0761 & -0.0915 & -0.7500 & 164.488 & 0.2854 & -0.1166 & -0.0647 & -0.7548 & 134.100 & 0.2843 \\
\hline 0.2 & -0.1122 & -0.1805 & -0.6599 & 94.615 & 0.2853 & -0.0997 & -0.1516 & -0.6676 & 99.378 & 0.2842 \\
\hline 0.1 & -0.0916 & -0.1094 & -0.7300 & 40.327 & 0.2845 & -0.1015 & -0.1575 & -0.6618 & 78.776 & 0.2846 \\
\hline-0.1 & $\begin{array}{l}-0.0968 \\
\end{array}$ & $\begin{array}{l}-0.1649 \\
\end{array}$ & -0.6736 & 26.865 & 0.2866 & $\begin{array}{l}-0.0988 \\
\end{array}$ & $\begin{array}{l}-0.1859 \\
-0.1859\end{array}$ & $\begin{array}{l}-0.6331 \\
\end{array}$ & 53.025 & 0.2860 \\
\hline-0.2 & -0.0973 & -0.1884 & -0.6497 & 22.500 & 0.2888 & -0.0986 & -0.1916 & -0.6274 & 44.791 & 0.2872 \\
\hline-0.3 & -0.0973 & -0.1996 & -0.6384 & 19.411 & 0.2920 & -0.0984 & -0.1955 & -0.6235 & 38.431 & 0.2890 \\
\hline-0.4 & -0.0973 & -0.2069 & -0.6310 & 17.042 & 0.2965 & -0.0983 & $\begin{array}{l}-0.1981 \\
\end{array}$ & $\begin{array}{l}-0.6208 \\
\end{array}$ & 33.452 & 0.2915 \\
\hline-0.5 & -0.0973 & -0.2120 & -0.6258 & 15.236 & 0.3029 & -0.0983 & -0.2000 & -0.6189 & 29.535 & 0.2952 \\
\hline \multicolumn{6}{|c|}{$T=500$} & & & & & \\
\hline$\phi_{1}$ & bias $\alpha$ & $\operatorname{bias} \rho_{1}$ & $\operatorname{bias} \rho_{2}$ & MSE & $\begin{array}{c}\text { Thiel's } \\
\text { U }\end{array}$ & & & & & \\
\hline 0.5 & -0.0966 & -0.1185 & -0.6892 & 650.965 & 0.2853 & & & & & \\
\hline 0.4 & -0.1024 & -0.1227 & -0.6850 & 431.069 & 0.2850 & & & & & \\
\hline 0.3 & -0.1031 & -0.1590 & -0.6486 & 316.506 & 0.2850 & & & & & \\
\hline 0.2 & -0.1030 & -0.1721 & -0.6355 & 242.632 & 0.2850 & & & & & \\
\hline 0.1 & -0.1027 & -0.1796 & -0.6280 & 191.968 & 0.2852 & & & & & \\
\hline - 0.1 & -0.1023 & -0.1875 & -0.6200 & 128.932 & 0.2858 & & & & & \\
\hline-0.2 & -0.1021 & -0.1897 & -0.6178 & 108.581 & 0.2863 & & & & & \\
\hline-0.3 & -0.1019 & -0.1912 & -0.6163 & 92.789 & 0.2871 & & & & & \\
\hline-0.4 & -0.1017 & -0.1923 & -0.6152 & 80.325 & 0.2882 & & & & & \\
\hline-0.5 & -0.1016 & -0.1931 & -0.6144 & 70.370 & 0.2898 & & & & & \\
\hline
\end{tabular}

